\title{
Determining the Potential Value of Salivary Plasmodium Falciparum Hisditine-Rich Protein 2 and Lactate Dehydrogenase as a Non-Invasive Test for Malaria
}

\section{FRANCIS OPOKU AGYAPONG ( $\square$ newopoku@yahoo.com )}

Komfo Anokye Teaching Hospital/Kwame Nkrumah University of Science and Technology https://orcid.org/0000-0002-3941-5591

\section{Daniel Ansong}

Department of Paediatrics, Kwame Nkrumah University of Science and Technology

\section{Alex Owusu-Ofori}

Department of Clinical Microbiology, Kwame Nkrumah University of Science and Technology

\section{Ruby Martin-Peprah}

Kwame Nkrumah University of Science and Technology y College of Agriculture and Natural Resources

\section{Millicent Opoku}

University of Ghana Noguchi Memorial Institute for Medical Research

Jewelna Akorli

University of Ghana Noguchi Memorial Institute for Medical Reseach

\section{Research}

Keywords: Malaria, PCR, Microscopy, Plasmodium, ELISA

Posted Date: January 27th, 2020

DOI: https://doi.org/10.21203/rs.2.21809/v1

License: (c) (i) This work is licensed under a Creative Commons Attribution 4.0 International License. Read Full License 


\section{Abstract}

\section{Background}

Malaria remains an important public health threat claiming many lives particularly in Sub-Saharan Africa. Light microscopy which is a blood-based test is the Gold standard for laboratory diagnosis of malaria in the clinical settings. The lack of sensitivity of Microscopy coupled with the challenges associated with blood sampling necessitates exploring alternative methods of identifying malaria cases.

Aims and Objectives

The aim of this study was to detect the presence of Plasmodium Lactate Dehydrogenase (pLDH) and Plasmodium falciparum Histidine-Rich Protein 2 (PfHRP2) in the saliva of suspected malaria patients and to compare the diagnostic accuracy of these saliva-borne biomarkers with the results of blood film microscopy using Nested PCR as the reference test.

\section{Methods}

The research was a comparative study. Matched saliva and blood samples of suspected malaria patients were collected. The blood samples were aliquoted into 2 smaller volumes and used to run blood film microscopy (thick and thin) and Nested PCR to detect DNA of the 5 Plasmodium species known to clinically infect man. Sand-wiched ELISA was separately run to qualitatively detect pLDH and PfHRP2 in the saliva samples. Accuracy indices of the saliva-based assays (saliva pLDH and PfHRP2) were compared with the conventional blood-based Microscopy.

Results

Of the participating 188 subjects, malaria prevalence rates of 51 (27.13\%), 80(42.55\%), $117(62.23 \%)$ and 95 (50.53\%) were detected by the Microscopy, saliva pLDH, saliva PfHRP2 and the PCR respectively. The sensitivity of the saliva PfHRP2 ELISA, 78.95\% (95\% Cl, 69.38-86.64\%) and saliva pLDH ELISA, $64.21 \%$ $(95 \% \mathrm{Cl} 53.72-73.79)$ were better than those obtained for the blood film Microscopy.

\section{Conclusions}

The saliva pLDH and the PfHRP2 ELISAs were found to be more sensitive $(64.21 \%$ and $78.95 \%$ respectively) in defining malaria cases than the results obtained for the conventional blood film microscopy. Both the saliva pLDH and the PfHRP2 ELISAs showed moderate agreement with the results of the Nested PCR with Kappa co-efficient of 0.44 and 0.5 respectively.

\section{Introduction}

Malaria is a life-threatening parasitic disease caused by some species of Plasmodium parasites that are transmitted through the bites of infected female Anopheles mosquitoes. In 2017, it was estimated that 
3.2 billion people - nearly half of the entire world's population - were at risk and that 91 countries and territories had ongoing malaria transmission [1]. WHO world malaria report, 2019 highlights there has not been significant progress in reducing global malaria cases over the past 4 years. In Ghana for instance, half a million more cases of malaria was recorded in 2018 compared to 2017 [2]. The burden of malaria still remains huge; in 2018 alone, 405,000 deaths due to malaria occurred worldwide [2]. Sub-Saharan Africa continues to carry a disproportionately high share of the global malaria burden. According to WHO World Malaria report in 2019, approximately $85 \%$ of all malaria deaths occurred in 20 countries all of which are in the Sub-Saharan Africa region except India. Nigeria and Ghana respectively accounted for $24 \%$ and $3 \%$ of all malaria deaths globally in the year 2018 [2].

In Ghana, a study revealed it costs households between US\$5.70 (for uncomplicated malaria) and US\$48.73 (for severe malaria) to treat malaria [3]. It is recorded that AngloGold Ashanti spent up to US\$55,000 every month on the treatment of malaria in its employees and their dependents and this excluded the valued loss in productivity as a result of absenteeism [4]. The total cost of malaria to businesses in Ghana was estimated in 2014 to be US\$6.58 million, $90 \%$ of which were direct costs [5]. It is therefore not surprising that, the 33 richest countries in the world are all malaria free [6].

Other studies have put forward that the economic benefits associated with significantly reducing or eliminating malaria are very huge. It is estimated that even with cost of investing in malaria elimination being very high (estimated US\$208.6 billion between 2016 and 2030, as well as another US\$673 million in research and development annually), the benefits to be harnessed in such an investment could be 40:1 worldwide and 60:1 in Sub-Saharan Africa. This means that, for every US\$1 invested in malaria eradication, economic gains of US\$40 worldwide and US\$60 in Sub-Saharan Africa will be made [7].

Among the 5 species of Plasmodium namely P. falciparum, P. malariae, P. ovale, P. vivax, P. knowlesi, which cause malaria, P. falciparum is known to cause the greatest morbidity and mortality.

Examination of Giemsa-stained thick and thin blood smears by light microscopy, a technique introduced in 1904, continues to be the standard for malaria diagnosis in most clinical settings. Microscopy as a modality of malaria diagnosis allows for detection and identification to the species level of all species, it makes room for parasite quantification and it can be used for monitoring parasite clearance following therapy.

Notwithstanding, microscopy as blood - based test is invasive and the pain associated with the needle prick in the course of drawing blood ward off patients. This could be challenging particularly in paediatric patients. Microscopy requires trained personnel and this could be a hindrance to rapid malaria diagnosis and treatment especially in human resource constrained settings. Carrying out blood film microscopy in settings without electricity as the case is in many rural areas in Sub-Saharan Africa could be very challenging. The use of needles in blood-based tests raises the risk of accidental transmission of infectious diseases. These techniques can also encounter difficulty with patient compliance particularly when blood collection is required from young children and in communities with cultural objections or blood taboos [8]. According to the Ghana Health Service Standard Treatment and Guidelines, the 
management of malaria requires initial test to confirm diagnosis and a repeated test post treatment to ensure parasite clearance. Such repeated blood sampling could complicate in phlebitis and cellulitis and this could have serious consequences especially in the immunocompromised such as diabetic and AIDS patients.

To circumvent the challenges associated with microscopy as a means of malaria diagnosis, researchers as well as clinicians have explored alternative diagnostic modality. The most outstanding of such alternative in malaria diagnostics is the introduction of Malaria (Plasmodium) antigen - based Rapid Diagnostic Test Kits (RDTs) in the mid - 1990s. RDTs detect parasite antigens from a small volume (usually 5 to $15 \mu \mathrm{L}$ ) of blood using an immunochromatographic assay impregnated on a test strip. The earliest RDTs employed primary antibodies to detect Plasmodium falciparum histidine-rich protein 2 (Pf HRP2) [9]. Today, commercial tests are tailored to local malaria epidemiology with different combinations of target antigens including genus-specific aldolases, and species-specific HRP2 and Plasmodium lactate dehydrogenase (pLDH). RDTs have proven to be very useful in rapid presumptive diagnosis of malaria especially in settings where blood film microscopy could not be easily carried out. However, RDTs also utilize blood and are not free from the challenges associated with blood - based tests. In contrast to blood, saliva presents a reduced biohazard [10] and can be painlessly collected in relatively large quantities by individuals with less training. Blood-borne biomarkers that cross from local vasculature into the salivary glands can in principle be detected in saliva [11]. Indeed, the diagnostic utility of saliva has been demonstrated in immunoassays for infectious diseases such as hepatitis, Ebola virus, measles, rubella, and HIV.

Potential biomarkers for malaria have also been identified in saliva [12]. The inconvenience associated with the traditional blood - based malaria test coupled with the low sensitivity of these tests as reported by many studies $[13,14,15,16]$ necessitates the exploration for alternative non-invasive potential sample for malaria diagnosis. This study therefore seeks to compare the diagnostic accuracy of saliva - borne Plasmodium falciparum histidine - rich protein 2 (PfHRP2) and Plasmodium Lactate Dehydrogenase (pLDH) with blood film microscopy in malaria case definition and advocate for the development of special RDTs with impregnated anti-bodies manufactured in a manner that can detect malaria antigens in saliva thereby overcoming the challenges associated with the traditional blood-based tests.

\section{Materials And Methods}

\subsection{Study Site and Study Population}

The research was a comparative study conducted at the Kumasi South Hospital (KSH), Biochemistry Laboratory of School of Medical Sciences, Kwame Nkrumah University of Science and Technology, Kumasi, Ghana and the Parasitology Department of the Noguchi Memorial Institute of Medical Research (NMIMR), Legon, Accra. NMIMR is the largest research institute in Ghana. KSH is the second largest hospital in the Ashanti region of Ghana located at the south of the regional capital Kumasi. The hospital serves as primary hospital and also referral centre for more than 1 million people in the Kumasi 
Metropolis and its adjoining towns. The study population included all patients seeking medical care at Kumasi South Hospital over a period of 1 month 1st to 30th October, 2017. The study is an expansion on our earlier work at same study site [13].

\section{Inclusion criteria}

Patients sent to the laboratory for malaria parasite investigation during the period of study with fever and/or history of fever within the last 3 days

\section{Exclusion criteria}

Patients who did not consent, those who were unable to produce saliva for instance young infants and those on current or past anti-malaria medication within the preceding 3 weeks (this was to reduce the effect of persistence of HRP2 antigen after parasite clearance)

\subsection{Sample Collection}

A total of 188 patients were recruited into the study. The temperature of each participant was checked and recorded against the assigned participant's Identity Number. About $2 \mathrm{mls}$ of venous blood sample was obtained from each participant by an expert phlebotomist in a blood sampling room. The blood sampling site; cubital fossa was rubbed with $70 \%$ alcohol and allowed to dry before drawing up the blood. Two (2) aliquots each of about $1 \mathrm{ml}$ in volume were made from the initially drawn blood sample and labeled as aliquots $A$ and $B$ for each participant. All aliquots $B$ were stored at $-20 \bowtie C$ for PCR detection of Plasmodium DNA later. Both thin and thick films were prepared from all aliquots labeled A for malaria parasite microscopy.

Saliva sample was collected from each participant. Participants were asked to rinse their mouths with water and then produce saliva into sterile tubes kept on ice to reduce biochemical processes such as enzyme action, protein degradation etc. $2-3 \mathrm{ml}$ of saliva was collected into sterile sputum containers and then aliquoted into 2 volumes of $1.5 \mathrm{mls}$ in separate sterile Eppendorff tubes and labeled as tube 1 and 2 for each participant's saliva sample. The saliva samples were stored at the hospital's laboratory at $-20^{\circ} \mathrm{C}$. The frozen saliva samples were transported to and thawed at the KNUST, School of Medical Sciences, Biochemistry Laboratory for ELISA.

\subsection{Blood Film Microscopy}

Blood aliquots from all participants labeled as A were used to prepare both thin and thick Giemsa stained slides for microscopic examination for malaria parasites. A drop of blood was placed on a clean greasefree specimen slide. Using the corner of a clean spreader slide, the drop of blood was spread in a circle of about $2 \mathrm{~cm}$ in diameter. The film was left to air-dry for 30 minutes and then staining was done with Giemsa solution (7.5\%, pH 7.2) for 30 minutes, after which the excess stain was washed off with buffered distilled water and then air-dried. Examination under the microscope with $X 100$ was done by an expert WHO certified microscopist for the presence or otherwise of Plasmodium parasite. A blood film was considered negative when no malaria parasites or trophozoites were observed after 100 high power fields 
(hpf) had been examined on the thick film. Where parasites were seen, they were counted against 200 white blood cells (WBCs). The parasite count per microliter ( $\mu \mathrm{l})$ of blood was obtained using the formula: (Parasite count/200WBC) $\times$ Absolute WBC count.

The thin film of each participant was prepared in parallel, a similar drop of blood from aliquot A of same participant was placed on clean grease-free specimen slide and a clean spreader slide was held at 45degree angle toward the drop of blood on the specimen slide such that the blood spread along the entire width of the spreader slide. The spreader slide was then pushed forward rapidly and smoothly still at the 45-degree angle. The blood smear was left to air dry for about 30 minutes and then fixed with $100 \%$ methanol. After complete drying, Giemsa staining was done as previously described and then subsequently observed under the microscope with $\mathrm{X} 100$ magnification by an expert WHO certified microscopist for Plasmodium species and stage of parasite identification.

For samples with high parasitaemia, $\geq 100$ parasites per high power field, parasite density was more accurately determined on the thin film. In the thin film, parasitized RBCs were counted against 1000 RBCs. The parasite count per microliter $(\mu \mathrm{l})$ was obtained using the formula: (parasitized RBCs $/ 1000$ RBCs) $\times$ Absolute RBC count.

\subsection{Enzyme-linked Immunosorbent Assay}

Anti-body sandwiched ELISA was run on each of the 2 saliva aliquots from each participant separately to detect Plasmodium falciparum Histidine-Rich Protein 2, Pf-HRP-2 and Plasmodium Lactate Dehydrogenase, $(\mathrm{pLDH})$ in the saliva samples.

Saliva Pf-HRP-2 ELISA was carried out on all saliva aliquots labelled 1 from each participant using Quantimal ${ }^{\text {TM }}$ CELISAP Ultra-sensitive Pf-HRP-2, (CELISA, Sydney, Australia) following the manufacturer's instruction. The results were read at $450 \mathrm{~nm}$ with an ELISA reader.

The Positive and Negative controls were supplied with the kit. The positive control was a culture supernatant containing standardised Pf-HRP-2 at a concentration of 100 nanogram/millilitre $(\mathrm{ng} / \mathrm{mL})$. Both the Positive and Negative controls were subjected to the same assay procedure on the same microtitre plate as described above.

Samples with absorbance readings greater than the absorbance reading +0.1 of the negative control were considered positive for Pf-HRP-2 as described by the manufacturer.

Similarly, saliva pLDH ELISA was carried out on all saliva aliquots labeled 2 from each participant using Quantimal ${ }^{\text {TM }}$ pLDH Malaria (CELISA, Sydney, Australia) following the manufacturer's instruction. The results were read with ELISA reader at $450 \mathrm{~nm}$. Samples with absorbance readings greater than the average of the absorbance readings for the two neagative control samples were considered positive for pLDH.

\subsection{Polymerase Chain Reaction}


A nested PCR adapted from $[13,17]$ was used targeting small subunit ribosomal RNA (18S rRNA) gene. The first PCR (Nest 1) involved the use of three primers (HUF, REV and PLF Table 1) which amplified a human gene and the Plasmodium genus. The human gene complex formed was UNR-HUF with a band size of 231basepairs (bp) which was expected in all samples irrespective of the presence or otherwise of Plasmodium

infection. That of the Plasmodium was UNR-PLF with band sizes between 783-821 bp depending on the species. In this PCR, a $20 \mu \mathrm{L}$ reaction of $1 \mathrm{X}$ OneTaq HotStart Standard Buffer, $0.075 \mu \mathrm{M}$ each of PLF and HUF, and $0.0125 \mu \mathrm{M}$ of HUF and $5 \mu \mathrm{L}$ of the extracted DNA and nuclease free water were used. The PCR was carried out at $94 \llbracket C$ for 3 minutes, followed by 35 cycles of $94 \llbracket C$ for 30 seconds, $58 \llbracket C$ for 1 minute, $68 \bowtie \mathrm{C}$ for 1 minute and then $68 \bowtie \mathrm{C}$ for 5 minutes.

The second PCR (Nest 2) detected Plasmodium specific species and incorporated the Nest 1 PCR products and primers for the various species (Table 1). In this PCR, a $25 \mu \mathrm{L}$ reaction of $1 \mathrm{X}$ OneTaq HotStart Standard Buffer (from New England Biolabs), $0.15 \mu \mathrm{M}$ each of NewPLFshort and falshort (for P. falciparum), $0.25 \mu \mathrm{M}$ Malshort, $0.1 \mu \mathrm{M}$ Vivshort, $0.36 \mu \mathrm{M}$ OvaNew and $0.1 \mu \mathrm{M}$ NewPKrev for P. malariae, P. vivax, P. ovale and P. Knowlesi respectively and $0.5 \mu \mathrm{L}$ of a 1 in 10 dilutions of Nest 1 PCR products. The PCR was then carried out at an initial $94 \llbracket C$ for 3 min, followed by 35 cycles of $94 \llbracket C$ for 30 seconds, $53 \otimes C$ for 1 minute, $68 \rrbracket C$ for 1 minute and then $68 \otimes C$ for 5 minutes. The PCR was done using a 2720 Thermal Cycler from Applied Biosystems. The PCR products were then run on a $2 \%$ agarose gel. After gel electrophoresis, the samples were visualized using a Toyobo Ultra-Violet (UV) transilluminator (from Japan). The PCR was considered positive in cases in which Plasmodium DNA was amplified by NEST 2 which was 1 in 10 dilutions of NEST 1 PCR products.

\subsection{Controls}

For NEST 1, the positive control employed DNA from cultured 3D7 strain (Plasmodium falciparum). Again, the human gene complex (UNR-HUF) present in each sample further served as positive control. For NEST 2, P. falciparum DNA sample obtained from human participants from another study and two samples of mixed P. falciparum and P. malariae also from another study provided by the Department of Immunology, Noguchi Memorial Institute for Medical Research, Ghana together with P. vivax DNA sample obtained from a laboratory in France were used as positive controls. The negative controls for both NEST 1 and NEST2 consisted of only the regents without DNA sample. 


\begin{tabular}{|c|c|c|c|c|c|}
\hline Name & Sequence $5{ }^{\prime}-3$ ', & Final conc $(\mu \mathrm{L})$ & $\operatorname{PCR}(T)$ & $\operatorname{Size}(b p)$ & Specificity \\
\hline PLF & AGTGTGTATCAATCGAGTTTC & 0.075 & $1^{\circ} \mathrm{PCR} 58^{\circ} \mathrm{C}$ & 783-821 & Plasmodium \\
\hline REV & GACGGTATCTGATCGTCTTC & 0.075 & $1^{\circ} \mathrm{PCR} 58^{\circ} \mathrm{C}$ & & Universal \\
\hline HUF & GAGCCGCCTGGATACCGC & 0.0125 & $1^{\circ} \mathrm{PCR} 58^{\circ} \mathrm{C}$ & 231 & Human \\
\hline NewPLFshort & CTATCAGCTTTTGATGTTAG & 0.15 & $2^{\circ} \mathrm{PCR} 53^{\circ} \mathrm{C}$ & & Plasmodium \\
\hline Malshort & TCCAATTGCCTTCTG & 0.25 & $2^{\circ} \mathrm{PCR} 53^{\circ} \mathrm{C}$ & 215 & P. malariae \\
\hline Falshort & GTTCCCCTAGAATAGTTACA & 0.15 & $2^{\circ} \mathrm{PCR} 53^{\circ} \mathrm{C}$ & 344 & P. falciparum \\
\hline Vivshort & AAGGACTTCCAAGCC & 0.10 & $2^{\circ} \mathrm{PCR} 53^{\circ} \mathrm{C}$ & 457 & P. vivax \\
\hline OvaNew & CCAATTACAAAACCATG & 0.36 & $2^{\circ} \mathrm{PCR} 53^{\circ} \mathrm{C}$ & 176 & P. ovale \\
\hline NewPKrev & CGCGGAGGCATC & 0.10 & $2^{\circ} \mathrm{PCR} 53^{\circ} \mathrm{C}$ & 389 & P. knowlesi \\
\hline
\end{tabular}

\subsection{Data analysis}

Data were entered into Microsoft Excel (2013), processed and analyzed using Statistical Package for Social Sciences (IBM SPSS Statistics, 2015).

To assess sensitivity and specificity, results of microscopy, Saliva pLDH ELISA and saliva PfHRP2 ELISA were compared with the Nested PCR results. The sensitivity was calculated as the proportion of positive test results obtained among samples scored as containing malaria parasites by the Nested PCR; the specificity was calculated as the proportion of negative test results obtained among samples whose PCR results were negative. Positive and negative predictive values were also calculated as the proportion of true-positive or true negative results among all samples scored as positive or negative by PCR, respectively. Kappa values, expressing the agreement beyond chance, were calculated. A Kappa ( $\mathrm{K})$ value of 0.01-0.20 indicates "slight agreement" a $\mathrm{k}$ value of 0.21-.04 depicts "fair agreement" a $\mathrm{k}$ value of $0.41-0.60$ indicates "moderate agreement" a $\mathrm{K}$ value of $0.61-0.80$ indicates "substantial agreement" and a $\mathrm{k}$ value of 0.81- 1.00 depicts "almost perfect or perfect agreement"

\section{Results}

Of the 188 participating patients, females 128 (68\%) out - numbered males $60(32 \%)$, majority of the participants, $50(26.60 \%)$ were within the age group 21-30 years whilst those greater than 70 years constituted the smallest age group $3(1.60 \%)$. The paediatric age group 1-10 years carried the greatest malaria burden by means of all the 4 tests/assays (Table 1 ). 
Majority of the participants 136 (72.35\%) were afebrile at time of participation (fever defined as axillary temperature of $37.5 \otimes \mathrm{C}$ or more) at the time of sampling whilst the remaining 52 recorded axillary temperature of $37.5^{\circ} \mathrm{C}$ or more. Out of the 52 febrile participants, 27 (51.92\%), 32(61.54\%), 38(73.08\%) and $37(71.15 \%)$ tested positive for malaria by means of Microscopy, saliva pLDH, saliva PfHRP2 ELISAs and PCR respectively. With the afebrile participants, 24(17.65\%), 48(35.29\%), 79(58.09\%) and 58(42.65\%) tested positive for malaria by means of Microscopy, saliva pLDH ELISA, saliva PfHRP2 ELISA and PCR respectively. All those microscopy (thin film) positive cases were Plasmodium falciparum. Similarly, all 95 (50.53\%) PCR positive cases fell on the P. falciparum band; no other species was detected.

\section{Comparison of the test/assay methods}

The PCR detected 45 of the microscopy positive cases as positive for P. falciparum and the remaining 6 were false positive. With the PCR results as the reference standard, the sensitivity and specificity of the microscopy tests were $47.37 \%(95 \% \mathrm{Cl}, 37.03 \%-57.88 \%)$ and $93.55 \%(95 \% \mathrm{Cl} 86.48 \%-97.60 \%)$ respectively. The negative predictive and the positive predictive values of Microscopy test with the PCR as the reference test were $65.03 \%(95 \% \mathrm{Cl}, 58.80 \%-67.96 \%)$ and $88.24 \%(95 \% \mathrm{Cl}, 77.07 \%-94.36 \%)$ respectively (Table 2)

The saliva Plasmodium Lactate Dehydrogenase (pLDH) ELISA detected 61 (64.21\%) of the PCR positive cases as positive whilst 19 (20.43\%) were falsely detected as positive. The sensitivity, specificity, negative predictive and positive predictive values of the saliva pLDH ELISA using the results of the PCR as reference standard were $64.21 \%(95 \% \mathrm{Cl}, 53.72-73.79 \%)$ 79.50\% (95\% Cl, 69.95-87.23\%), 68.52\% (95\% $\mathrm{Cl}, 62.00-74.38)$ and $76.25 \%(95 \% \mathrm{Cl}, 67.66-83.13 \%)$ respectively (Table 2). The saliva Plasmodium falciparum Histidine-Rich Protein 2 (PfHRP 2) ELISA detected 75 (78.95\%) of the positive PCR cases also as positive. With the PCR results as reference test, the sensitivity, specificity, negative predictive and positive predictive values of the saliva PfHRP2 ELISA were 78.95\% (69.38-86.64\%) $54.84 \%(95 \% \mathrm{Cl}$, 44.17-65.19\%), 71.83\% (95\% Cl, 62.37-79.69\%) and 64.10\% (95\% Cl, 58.25 69.57\%) respectively (Table 2).

\section{Discussion}

The current study highlights the presence of diagnostically utilizable Plasmodium antigens in saliva samples of malarial patients and calls for further exploration of saliva as a potential sample for malaria diagnostics.

Compared to PCR, the saliva pLDH and the PfHRP2 ELISAs were found to be more sensitive $64.21 \%$ (95\% $\mathrm{Cl}, 53.72-73.79 \%)$ and $78.95 \%(95 \% \mathrm{Cl}, 69.38-86.64 \%)$ respectively, than the results obtained for the microscopy. Just like the microscopy, both the saliva pLDH and the PfHRP2 ELISAs showed moderate agreement with the Nested PCR (Kappa 0.44 and 0.50 respectively). The Saliva assays were however, less specific than the Microscopy.

In a similar study in the Philippines reported that compared to Microscopy, saliva PfHRP2 was both 100\% sensitive and specific in defining malaria cases [10] In yet another study in Ghana, the sensitivity of 
Saliva PfHRP2 ELISA in defining malaria cases was reported as $43 \%$ when compared to microscopy [18].

With the study in Philippines, the small sample size of 8 might explain the higher sensitivity and specificity obtained compared to those accuracy indices reported by this study. The Ghanaian study on the other hand utilized the supernatant of spun saliva in their analysis unlike this study that employed whole saliva. Spinning and subsequent utilization of the supernatant might have lowered the sensitivity. This might explain the significant difference between the levels of sensitivity reported by this study and the previous Ghanaian study.

For the Saliva pLDH ELISA, the sensitivity of $64.21 \%$ reported by this study is lower than the $77.9 \%$ obtained with whole saliva but higher than $48.40 \%$ obtained with saliva supernatant in a similar study in Nigeria [19]. The correlation co-efficient $(\mathrm{K}=0.48)$ reported the Nigerian study [19] for whole saliva pLDH is comparable to that reported by this study $(\mathrm{K}=0.44)$. However, the correlation co-efficient for the saliva supernatant pLDH results with respect to Microscopy results $(K=0.21)$ was weaker than that reported by this study. In a similar study in Kenya [20], when the sensitivity of saliva pLDH was compared with blood film microscopy, saliva pLDH was found to be only $35 \%$ sensitive with weak positive correlation relative to the microscopy results.

The varying level of sensitivity among the various studies may be due to the design and quality of the assay kit and the assay procedure as different assay kits from different manufacturers were used. Also, lack of standardized technique of saliva sample handling and storage conditions might explain the difference in the results reported. Again, both the Nigerian and Kenyan studies did not use molecular techniques like the PCR as the reference test in obtaining their accuracy indices.

It is unclear why the level of sensitivity of the PfHRP2-based ELISA was higher but less specific than the pLDH-based ELISA. This might be due to the presence of other proteins or substances in saliva samples that cross-reacted with the anti - bodies employed against the PfHRP2 antigen. Again, PfHRP2 may persist in circulation up to 28 days in individuals whom malaria has been successfully treated. On the other hand, pLDH is secreted only by live parasites and is quickly cleared from the circulation. Detection of pLDH is therefore more suggestive of active parasitaemia.

The role of ELISA in malaria research and epidemiological surveys cannot be over-emphasized. But clinically, it may be impractical to use ELISA to diagnose malaria, because aside requirement for expertise, patients will have to be batched into groups thereby increasing turn - around time. However, the increasing detection of these potential malaria diagnostic biomarkers in other non - blood body fluids like saliva should eventually stimulate the development of lateral flow chromatographic assays that could be used for self - diagnosis of malaria just like the Oral - Quick HIV diagnostic kit that utilizes saliva making self-diagnosis of HIV possible even at home.

\section{Conclusion}


All 3 tests/assays; Microscopy, saliva pLDH and saliva PfHRP2 ELISAs showed moderate correlation with the nested PCR. The saliva pLDH and the PfHRP2 ELISA were found to be more sensitive $(64.21 \%$ and $78.95 \%$ respectively) in defining malaria cases than the results obtained for the Microscopy. The Saliva assays were however, much less specific than the Microscopy.

\section{Recommendations}

Given the comparable results of the saliva-based assays relative to the conventional blood-based malaria tests, it is recommended that further multi - centred studies are done on saliva with much greater sample size.

Because of the limitation of using ELISA in the clinical settings as patients will have to batched thereby increasing turn-around times. Lateral flow immunochromatography kits with multiple anti-bodies to more than one of these Plasmodium antigens that utilize saliva as sample may be developed and tried to serve as non- invasive screening tests for malaria.

\section{Declarations}

\section{Consent for publication}

All authors read and approved the final manuscript for publication.

\section{Ethical approval}

The authors declare that all aspects of the study were examined and approved by the Committee on Human Research, Publication and Ethics (CHRPE) School of Medical Sciences of the Kwame Nkrumah University of Science and Technology (KNUST) and KATH in Kumasi, Ghana.

Conflict of interest: We declare that we have no conflict of interest.

Availability of data and material: All and material pertaining to this work could be obtained via email from the authors or from malaria journal repository on request.

Funding: The research was not funded

Authors' contribution: This work was carried out in collaboration among all authors. Author FOA helped with study conception, design, data analysis and writing of the manuscript. Authors FOA, MO, JA and GMA helped with the field work. Authors DA, RMP and AOO helped with study supervision and critical revision of the manuscript for intellectual content. DA and RMP provided logistics for the study. All authors read and approved the final manuscript for publication.

Acknowledgement: The authors express their gratitude to the management of Kumasi South Hospital, Kumasi for allowing their facility and their patients to be used for this study. The role of the laboratory 
personnel of the Molecular Biology Department of the Kwame Nkrumah University of Science and Technology, Kumasi is very much appreciated. The authors also express their gratitude management of Noguchi Memorial Institute for Medical Research particularly the staff of Parasitology Department for allowing their facility to be used for the DNA extraction and the PCR procedures.

\section{References}

1. WHO, World Malaria Report. Geneva: World Health Organization, 2018

2. WHO, World Malaria Report. Geneva: World Health Organization, 2019

3. Sicur E., Vieta A., Lindner L., Constenla D. \& Sauboin C. (2013). The Economic Cost of Malaria in Children in Three Sub-Saharan African Countries: Ghana, Tanzania and Kenya. Malaria Journal; 12:307

4. Ghana: Malaria Control Programme - AngloGold Ashanti, 2017 Report

5. Nonvignon J., Aryeetey G.C., Malm K.L., Agyemang S.A., Aubyn V.N.A, Peprah N.Y., Bart-Plange C.N, \& Aikins M. (2016). Economic burden of malaria on businesses in Ghana: a case for the private investment in malaria control. Malaria Journal Sep 6, 2016 15:454

6. Gallup J.L. \& Sachs J.D. (2001) The economic burden of malaria. American Journal of Tropical Medicine and Hygiene. 64: 85-96.

7. Action and investment to defeat malaria 2016-2030. Geneva: Roll Back Malaria Partnership; 2015.

8. Nokes D.J., Enquselassie F., Nigatu W., Vyse A.J, Cohen B.J., Brown D.W.G. \& Cutts F.T. (2001): Has oral fluid the potential to replace serum for the evaluation of population immunity levels? A study of measles, rubella and hepatitis B in rural Ethiopia. Bulletin of the World Health Organization: 79(7):588-595.

9. Shiff C.J., Minjas J. \& Premji Z. (1994). The ParaSight-F test: a simple rapid manual dipstick test to detect Plasmodium falciparumParasitology Today 10(2): 494-495.

10. Fung A.O., Damoiseaux R., Grundeen S., Panes J.L., Horton D.H., Judy J.W. \& Moore T.B. (2012) Quantitative detection of PfHRP2 in saliva of malaria patients in Philippines. Malaria Journal. 2012; 11: 175 .

11. Haeckel R. \& Hanecke P. (1996). Application of saliva for drug monitoring. An in vivo model for transmembrane transport. European Journal of Clinical Chemistry and Clinical Biochemistry. 34(3): 171-191. 5

12. Formenty P., Leroy E.M., Epelboin A, Libama F., Lenzi M., Sudeck H., Yaba P., Allarangar Y., Boumandouki P., Nkounkou V.B., Drosten C., Grolla A., Feldmann H. \& Roth C. (2006). Detection of Ebola virus in oral fluid specimens during outbreaks of Ebola virus hemorrhagic fever in the Republic of Congo. Clinical Infectious Diseases 42: 1521-1526.

13. Agyapong F.O., Ansong D., Owusu-Ofori A. \& Martin-Peprah R. (2019) Diagnostic Performance of Blood Film Microscopy and PfHRP2-based RDT in a Routine Clinical Setting of a Secondary Health Facility in Ghana. Journal of Advances in Medicine and Medical Research 31(4): 1-9 
14. Golassa L, Enweji N, Erko B, Assefa A, Swedberg G. (2013) Detection of a substantial number of submicroscopic Plasmodium falciparum infections by Polymerase chain reaction: A potential threat to malaria control and diagnosis in Ethiopia. Malaria Journal 2013;12:352.

15. Laban M.N., Kobayashi T., Hamapumbu H., Sullivan D., Mharajurwa S., Thuma P.E., Shiff C.J. \& Moss W.J. (2015) Comparison of a PfHRP2-based rapid diagnostic test and PCR for malaria in a low prevalence setting in rural southern Zambia: Implications for elimination. Malaria Journal 2015; 14:25.

16. Batwala V., Magnussen P. \& Nuwaha F. (2010) Are rapid diagnostic tests more accurate in diagnosis of Plasmodium falciparum malaria compared to microscopy at rural health centres? Malaria Journal 2010;9:349.

17. Miguel-Oteo M., Jiram A.I., Ta-Tang T.H., Lanza M., Hisam S. \& Rubio J.M. Nested Multiplex PCR for identification and detection of human Plasmodium species including Plasmodium knowlesi. Asian Pacific Journal of Tropical Medicine 2017;10(3):299-304

18. Wilson M.L. (2013). Laboratory Diagnoses of Malaria. Conventional and Rapid Diagnostic Methods. Archives of Pathology and Laboratory Medicine 13796): 805-11

19. GbotoshoO., Happi C.T., Folarin O., Keyamo O., Sowunmi A. \& Oduola A.M.J. (2010) Rapid Detection of Lactate Dehydrogenase and Genotyping of Plasmodium falciparum in Saliva of Children with Acute Uncomplicated Malaria American Journal of Tropical Medicine and Hygiene. 2010 Sep; 83(3): 496-501.

20. Nambati E.A., Kiarie W.C., Kimani F., Kimotho J.H., Otinga M.S., Too E., Kaniaru S., Limson J. \& Bulimo W. (2018) Unclear association between levels of Plasmodium falciparum lactate dehydrogenase ( $\mathrm{pfLDH}$ ) in saliva of malaria patients and blood parasitaemia: diagnostic implications? Malaria Journal Jan 5; 17(1):9.

\section{Tables}

Due to technical limitations, Tables 1-2 are provided in the Supplementary Files section.

\section{Supplementary Files}

This is a list of supplementary files associated with this preprint. Click to download.

- rawdataAgyapong.xlsx

- Tables.docx

- rawdataAgyapong.xlsx

- Tables.docx 